\title{
Monoacylglycerol lipase inhibitor JZL184 regulates apoptosis and migration of colorectal cancer cells
}

\author{
MUYUAN MA ${ }^{1,2}$, JIE BAI $^{1}$, YE LING $^{1}$, WEILONG CHANG ${ }^{1}$, \\ GENGCHEN XIE $^{1}$, RUIDONG LI ${ }^{1}$, GUOBIN WANG ${ }^{1}$ and KAIXIONG TAO ${ }^{1}$ \\ ${ }^{1}$ Department of Gastrointestinal Surgery II, Union Hospital, \\ Tongji Medical College of Huazhong University of Science and Technology, Wuhan, Hubei 430022; \\ ${ }^{2}$ Department of Surgical Oncology, General Hospital of Ningxia Medical University, Yinchuan, Ningxia 750004, P.R. China
}

Received February 8, 2015; Accepted December 11, 2015

DOI: $10.3892 / \mathrm{mmr} .2016 .4829$

\begin{abstract}
Monoacylglycerol lipase (MAGL) is involved in the degradation of triacylglycerol. Previous studies have demonstrated that MAGL regulates tumor growth and metastasis via fatty acid networks, and is associated with colorectal cancer. JZL184 is a MAGL inhibitor, which in the present study was administered to colorectal cancer cell lines, resulting in decreased tumor proliferation, increased apoptosis and increased tumor cell sensitivity to 5-fluorouracil. B-cell lymphoma 2 (Bcl-2) and Bcl-2-associated $\mathrm{X}$ protein (Bax) are key proteins in apoptosis. The expression levels of Bcl-2/Bax were determined in colorectal cancer cell lines following JZL184 administration, and it was observed that the mRNA and protein expression levels of $\mathrm{Bcl}-2$ were decreased, whereas the expression levels of Bax were increased. These results indicated that JZL184 may induce tumor cell apoptosis by regulating the expression of Bcl-2 and Bax. Epithelial-mesenchymal transition (EMT) is closely associated with metastasis. Administration of JZL184 in various malignant colorectal cancer cell lines suppressed migration and altered the expression of EMT markers; E-cadherin was increased, whereas the expression levels of vimentin and zinc finger protein SNAI1 were decreased. These results suggested that JZL184 was able to regulate the EMT process, in order to control the migration of colorectal cancer cells, particularly in tumors with a stronger metastatic capability. Therefore, in colorectal cancer, MAGL may be considered a potential therapeutic target and JZL184 may be a possible therapeutic agent.
\end{abstract}

Correspondence to: Professor Kaixiong Tao, Department of Gastrointestinal Surgery II, Union Hospital, Tongji Medical College of Huazhong University of Science and Technology, 1277 Jiefang Road, Wuhan, Hubei 430022, P.R. China

E-mail: tao_kaixiong@163.com

Key words: monoacylglycerol lipase, JZL184, colorectal cancer, apoptosis, epithelial-mesenchymal transition

\section{Introduction}

Monoacylglycerol lipase (MAGL), which is an enzyme of the serine hydrolase family, is the key enzyme associated with the degradation of triacylglycerol (TAG) into free-fatty acids (FFA) (1). MAGL hydrolyzes 2-arachidonoylglycerol (2-AG) into arachidonic acid and glycerol, thus regulating the endocannabinoid system (2). A previous study regarding MAGL focused on the role of 2-AG hydrolysis in endocannabinoid signaling (3). 2-AG belongs to the endocannabinoid system, which is associated with the regulation of inflammation, nociception and the immune response (4), and exerts a neuroprotective effect in brain injury (5). Long et al (6) identified a selective and effective inhibitor of MAGL, JZL184, which significantly decreased the hydrolytic activity of MAGL and inhibited 2-AG hydrolysis in the brains of mice. It has previously been demonstrated that MAGL is highly expressed in certain types of aggressive cancer, and regulates the fatty acid network to influence tumor metastasis (7). Furthermore, Nomura et al (8) demonstrated that in androgen-dependent prostate cancer, MAGL influenced tumor proliferation and metastasis via the cannabinoid system and fatty acid network. As a characteristic of the malignant colorectal cancer phenotype, metastasis is markedly associated with the epithelial-mesenchymal transition (EMT) process (9). A previous study indicated that MAGL is a marker of EMT; therefore, the effects of JZL184 in colorectal cancer have recently garnered interest (10). Furthermore, Ye et al (11) observed that MAGL was highly expressed in colorectal cancer tissues, as compared with normal tissue, and MAGL expression was associated with the body mass index (BMI) of patients. With decreasing MAGL activity, the protein expression levels of cyclin D1 and B-cell lymphoma 2 (Bcl-2) were reduced, apoptosis was increased, and the cell cycle was arrested in colorectal cancer cell lines (11). Metastasis and decreased apoptosis are associated with tumorigenesis, both of which are particularly associated with Bcl-2/Bcl-2-associated X protein (Bax) and the EMT process. A previous study demonstrated that Bcl-2/Bax was closely associated with apoptosis in colorectal cancer pathogenesis $(12,13)$. Bcl-2 decreases apoptosis in cancer cells (14); however, Bax inhibits the function of Bcl-2 in order to induce cell death (15). However, the association 
between JZL184 and Bcl-2/Bax in colorectal cancer remains to be elucidated.

The present study aimed to determine the effects of JZL184 on apoptosis and migration, and to elucidate the association between JZL184, Bcl-2/Bax and EMT markers.

\section{Materials and methods}

Cell culture. The HCT116, SW480 and LoVo colorectal cancer cell lines were supplied by the Laboratory of Gastrointestinal Surgery, Union Hospital (Wuhan, China). The cells were grown in high glucose Dulbecco's modified Eagle's medium (DMEM; Hyclone; GE Healthcare Life Sciences, Logan, UT, USA) supplemented with $10 \%$ fetal bovine serum (FBS; Gibco; Thermo Fisher Scientific, Inc., Waltham, MA, USA). SW620 cells (supplied by the Laboratory of Gastrointestinal Surgery, Union Hospital) were grown in RPMI-1640 (Hyclone; GE Healthcare Life Sciences) and supplemented with $10 \%$ FBS (16). All cells were maintained in a humidified $37^{\circ} \mathrm{C}$ incubator containing 5\% $\mathrm{CO}_{2}$. The HCT116, SW480 and LoVo cell lines were grown in DMEM with varying concentrations of JZL184 (10, 25 or $50 \mu \mathrm{M}$; Sigma-Aldrich, St. Louis, MO, USA) or dimethyl sulfoxide (DMSO; $0.1 \%$ ), representing the control group, for $48 \mathrm{~h}$. These cells were then used in the following assays, except the migration assay.

3-(4,5-dimethylthiazol-2-yl)-2,5-diphenyltetrazolium bromide (MTT) assay. Cells $\left(1 \times 10^{4}\right)$ of the HCT116, SW480 and LoVo cell lines were grown in 96-well plates for $48 \mathrm{~h}$, as described. Medium was then replaced in the cell cultures with DMEM only (control group) or DMEM supplemented with $10 \mu \mathrm{M}$ JZL184 (experimental group). Cells were then treated with varying concentrations of 5-fluorouracil $(10,100,200$ or $500 \mu \mathrm{M})$ for $48 \mathrm{~h}$. Subsequently, $20 \mu \mathrm{l}(5 \mathrm{mg} / \mathrm{ml})$ MTT was added to each well for $4 \mathrm{~h}$. The MTT formazan precipitate was dissolved in DMSO to $150 \mu \mathrm{l}$. The absorbance of each well was measured at a wavelength of $490 \mathrm{~nm}$, and the cell proliferation inhibition rate was calculated using the following formula: Proliferation inhibition rate $=[1-$ value of the experimental group/average value of the control (5-fluorouracil only) group] x 100\% (17).

Annexin-V allophycocyanin (APC)/propidium iodide (PI) double labeling. The cells were collected and washed with phosphate-buffered saline (PBS). According to the manufacturer's protocol, samples were stained with Annexin-V APC (Nanjing KeyGen Biotech Co., Ltd., Nanjing, China) and PI (Sigma-Aldrich) for $30 \mathrm{~min}$ at room temperature in the dark. The cells were then examined using a BD FACSCanto II flow cytometer (BD Biosciences, Franklin Lakes, NJ, USA).

Reverse transcription-quantitative polymerase chain reaction $(R T-q P C R)$. Total mRNA was extracted from the cells using TRIzol $^{\circledR}$ (Takara Bio, Inc., Otsu, Japan). According to the manufacturer's protocols the RNA was reverse transcribed to cDNA using Primescript RT Master mix (Takara Bio, Inc.). RT-qPCR was used to detect the levels of mRNA in each sample, with glyceraldehyde-3-phosphate dehydrogenase (GAPDH) serving as an internal control. SYBR Green Master mix (Takara Bio, Inc.) and the StepOnePlus ${ }^{\mathrm{TM}}$ Real-time PCR system (Applied Biosystems; Thermo Fisher Scientific, Inc.) were used to conduct qPCR, according to the manufacturers' protocols. The qPCR cycling conditions were as follows: Denaturing the cDNA at $95^{\circ} \mathrm{C}$ for $30 \mathrm{sec}$; annealing at $60^{\circ} \mathrm{C}$ for $60 \mathrm{sec}$; and extension at $95^{\circ} \mathrm{C}$ for $5 \mathrm{sec}$. The reaction proceeded for 40 cycles, gathering the data of each sample, and the mRNA expression levels were evaluated using the $2^{-\Delta \Delta \mathrm{Cq}}$ method (18). The primers were obtained from Genscript Corporation (Nanjing, China) and the sequences were as follows: Bcl-2, forward 5'-TTGCCCTCAAACAGAACAGC-3' and reverse 5'-TGCAGCTCCTCTTGGCTAAA-3'; Bax, forward 5'-GGC CGGGTTGTCGCCCTTTT-3' and reverse 5'-CCGCTCCCG GAGGAAGTCCA-3'; and GAPDH, forward 5'-GTTCCCACT GTCGATGTCTCA-3' and reverse 5'-CCCTTCATCTTGCCC TCAGA-3'.

Western blot analysis. Cells were lysed with sodium dodecyl sulfate-polyacrylamide gel electrophoresis (SDS-PAGE) Sample Loading buffer (Beyotime Institute of Biotechnology, Haimen, China) supplemented with proteinase inhibitors (Roche Diagnostics, Basel, Switzerland), and the concentration of total protein was measured using a Bradford Protein assay kit (Beyotime Institute of Biotechnology). Proteins $(5 \mu \mathrm{g} / \mu \mathrm{l})$ were separated on 8 or $12 \%$ SDS-polyacrylamide gels by electrophoresis and were transferred to polyvinylidene fluoride (PVDF) membranes (EMD Millipore, Billerica, MA, USA). The PVDF membranes were blocked with 5\% skimmed milk protein and $0.1 \%$ Tween 20 for $1 \mathrm{~h}$. Primary antibodies were added and incubated overnight at $4^{\circ} \mathrm{C}$, prior to the addition of goat anti-rabbit secondary antibody for $2 \mathrm{~h}$ at room temperature. The primary antibodies used in the present study, from Cell Signalling Technology Inc. (Danvers, MA, USA) and used at a dilution of 1:1,000, were: Rabbit anti-E-cadherin (cat. no. 3195), rabbit anti-vimentin (cat. no. 5741), rabbit anti-zinc finger protein SNAI1 (Snail; cat. no. 3879), rabbit anti-Bcl-2 (cat. no. 2870), rabbit anti-Bax (cat. no. 2772). Rabbit anti-GAPDH was supplied by Sigma-Aldrich (cat. no. G9545) and used at a dilution of 1:5,000. The secondary antibody used was polyclonal horseradish peroxidase-conjugated goat anti-rabbit IgG (dilution, 1:1,000; cat. no. BA1054; Boster Biological Technology, Pleasanton, CA, USA). Background autofluorescence was removed and blots were imaged using a ChemiDoc XRS+ imaging system and blots were analyzed with Image Lab software (Bio-Rad Laboratories, Inc., Hercules, CA, USA), with GAPDH acting as a reference protein. Relative expression of the relevant protein was calculated using the formula: Relative expression $=$ (density value of the experimental group - value of GAPDH) / (density value of the control group - value of GAPDH).

Cell migration assay. SW480 and SW620 cells $\left(1 \times 10^{6}\right)$ were treated with $10 \mu \mathrm{M}$ JZL184 for $48 \mathrm{~h}$ and plated onto transwell cell culture inserts ( $8 \mu \mathrm{m}$ microporous filters; BD Biosciences), which provided an artificial basement membrane, for $12 \mathrm{~h}$. The upper chamber of the transwell system contained DMEM (in the case of SW480 cells) or RPMI-1640 (for SW620 cells); the lower chamber contained corresponding medium supplemented with $20 \%$ FBS. The migrating cells were stained with $0.1 \%$ crystal violet and five randomly selected areas were used to count the number of cells using an Olympus 1X71 inverted microscope (Olympus Corporation, Tokyo, Japan) (16). 

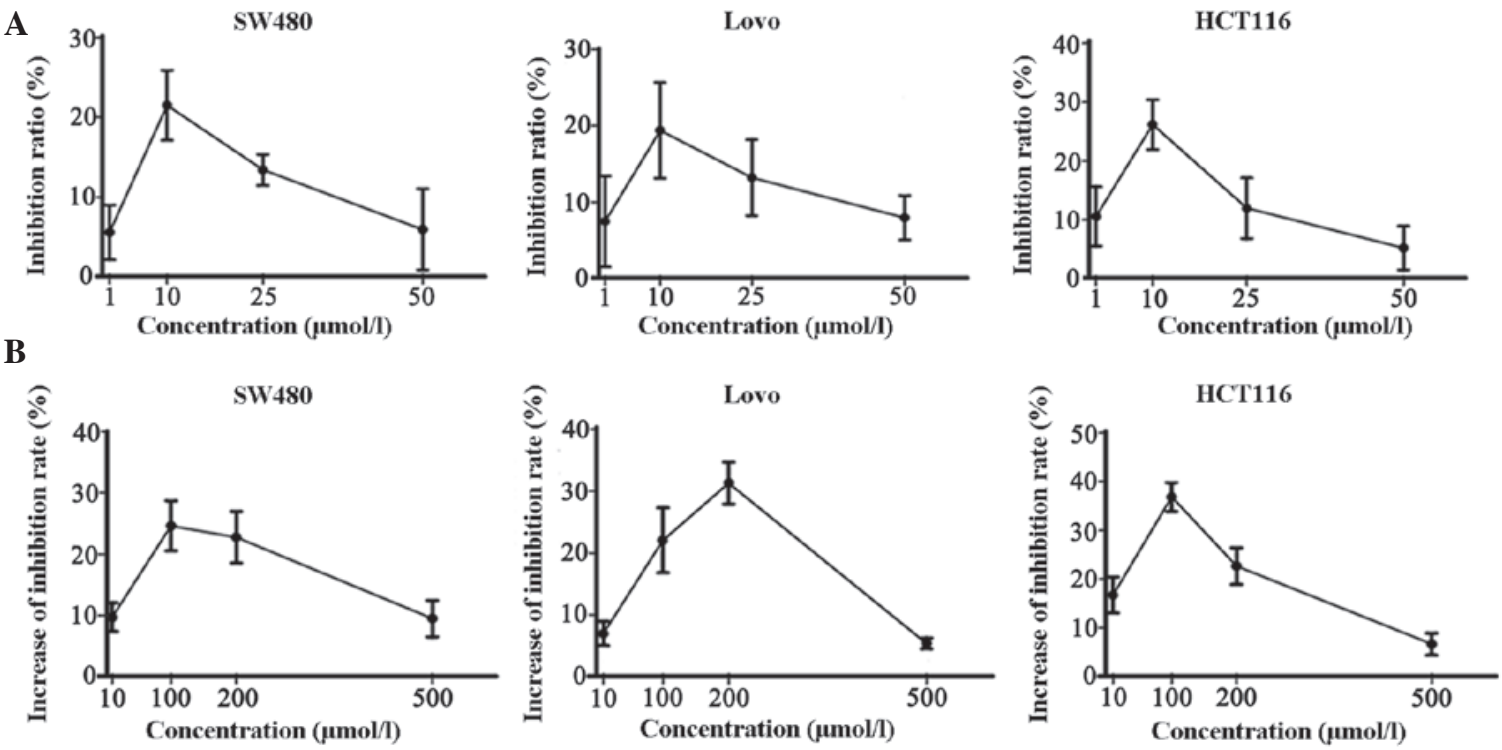

Figure 1. JZL184 suppressed the proliferation of colorectal cancer cells. (A) As assessed by 3-(4,5-dimethylthiazol-2-yl)-2,5-diphenyltetrazolium bromide assay, proliferation decreased by varying degrees in response to various concentrations of JZL184 in three colorectal cancer cell lines. Cell proliferation was decreased the most following treatment with $10 \mu \mathrm{M}$ JZL184 in each group. Dimethyl sulfoxide served as the control. (B) JZL184 (10 $\mu$ M) combined with various concentrations of 5-fluorouracil, as indicated on the $x$-axis, was administered to the tumor cells for $48 \mathrm{~h}$. The inhibition ratios of cancer cells were increased in cells treated with both JZL184 and 5-fluorouracil, as compared with 5-fluorouracil alone. Data are presented as the mean \pm standard deviation.

Statistical analysis. Each experiment was performed at least three times independently. The data are presented as the mean \pm standard deviation. SPSS v. 18.0 software (SPSS, Inc., Chicago, IL, USA) was used for all statistical analyses. Student's t-test was used to statistically analyze the data. $\mathrm{P}<0.05$ was considered to indicate a statistically significant difference.

\section{Results}

JZL184 influences the proliferation of colorectal cancer cell lines. JZL184 was dissolved in DMSO and administered to the SW480, LoVo and HCT116 colon cancer cell lines. DMSO was used as a negative control. After a $48 \mathrm{~h}$ incubation, the inhibition ratio was measured by MTT assay. The inhibition ratio was non-significantly increased $(\mathrm{P}>0.05)$ in all three cell lines following treatment with $10 \mu \mathrm{M}$ JZL184 (Fig. 1A). In addition, JZL184 $(10 \mu \mathrm{M})$ was combined with various concentrations of 5-fluorouracil. In the colorectal cancer cells treated with JZL184, cell growth was decreased $(\mathrm{P}>0.05)$. The inhibition ratio was increased in the cells treated with both JZL184 and 5 -fluorouracil, as compared with in the cells treated with 5-fluorouracil alone (Fig. 1B; P>0.05).

JZL184 induces colorectal cancer cell apoptosis. The present study demonstrated that JZL184 had marked effects on the proliferation of colorectal cancer cell lines. Proliferation is closely associated with apoptosis in cancer; therefore, apoptosis of the colorectal cancer cells treated with JZL184 was investigated by flow cytometry. Compared with the control group, the number of cells in early (Q2) and late (Q4) apoptosis was increased in the treatment group (Fig. 2A). The rate of apoptosis was increased in the three colorectal cancer cell lines (Fig. 2B). These results indicate that JZL184 may induce apoptosis via decreasing proliferation in colorectal cancer cell lines. In addition, the expression levels of MAGL were significantly decreased following treatment with JZL184 (Fig. 2C and D).

JZL184 regulates Bcl-2 and Bax to induce apoptosis of colorectal cancer cell lines. JZL184 promoted the apoptosis of colorectal cancer cells. Bcl-2 and Bax are closely associated cell apoptosis proteins (19); therefore, the effects of JZL184 on their mRNA expression levels were detected by RT-qPCR. The mRNA expression levels of Bcl-2 were significantly lower in the JZL184 intervention groups, as compared with the control group; however, the expression levels of Bax were significantly higher following JZL184 administration, as compared with in the control group in the three colorectal cancer cell lines (Fig. 3A). The protein expression levels of Bcl-2 and Bax were also detected following JZL184 administration. Consistent with the mRNA expression levels, following JZL184 intervention Bcl-2 protein expression levels were significantly decreased, as compared with the control group, whereas Bax protein expression levels were increased (Fig. 3B and C). These results indicate that JZL184 is able to regulate apoptosis via altering the mRNA and protein expression of Bcl-2 and Bax.

JZL184 decreases migration in colorectal cancer cell lines. Migration is required for metastasis in colorectal cancer. To investigate whether JZL184 affected the migratory ability of colorectal cancer cells, SW480 and SW620 cells were selected. SW480 cells were initially derived from colon cancer tissue from a patient with Duke's type B colon cancer, whereas SW620 cells were initially derived from the metastatic lymph nodes of a patient with Duke's type C colon cancer, as listed by the American Type Culture Collection (www.lgcstandards-atcc.org/). The metastatic potential of these cells from two stages of colorectal cancer differs (20). Following administration of JZL184, the migra- 
$\mathbf{A}$
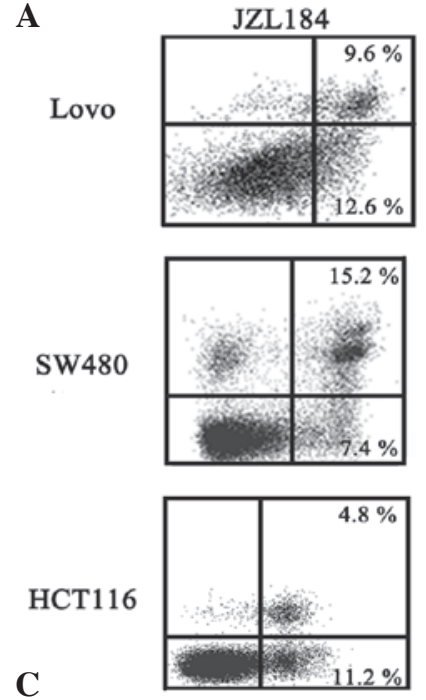

C

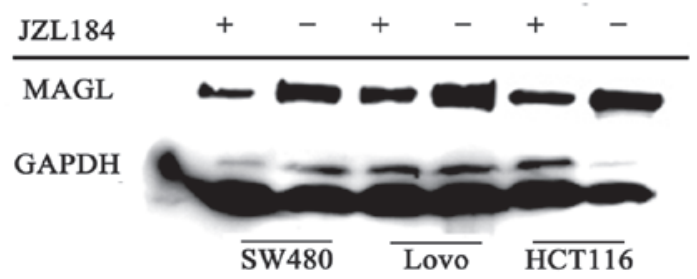

B

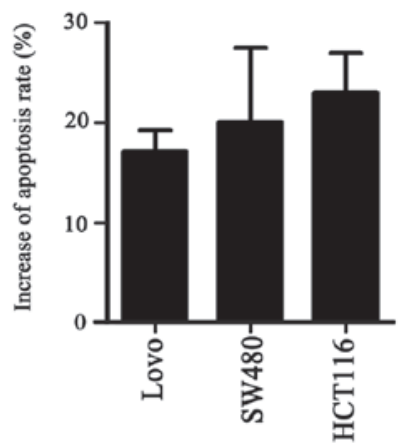

$\mathbf{D}$

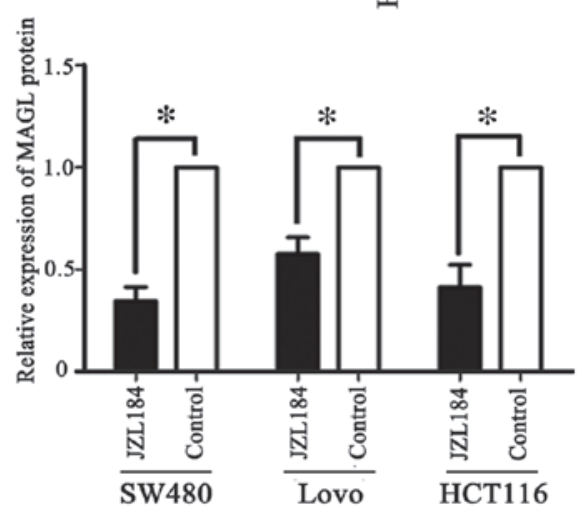

Figure 2. JZL184 induced apoptosis of colorectal cancer cells and regulated the expression of MAGL. (A) Apoptosis was detected using flow cytometry. The number of apoptotic LoVo, HCT116 and SW480 cells was increased. (B) Compared with the control group, the apoptotic rate was increased in the three colorectal cancer cell lines to $17.11 \pm 4.77 \%$ (LoVo), 20.04 $\pm 7.43 \%$ (SW480) and 22.98 $\pm 8.89 \%$ (HCT116). (C and D) As assessed by western blotting, the expression levels of MAGL following JZL184 treatment were significantly decreased in the three colorectal cancer cell lines, as compared with in the control group. Data are presented as the mean \pm standard deviation. ${ }^{P}<0.05$. MAGL, monoacylglycerol lipase; GAPDH, glyceraldehyde-3-phosphate dehydrogenase.

A

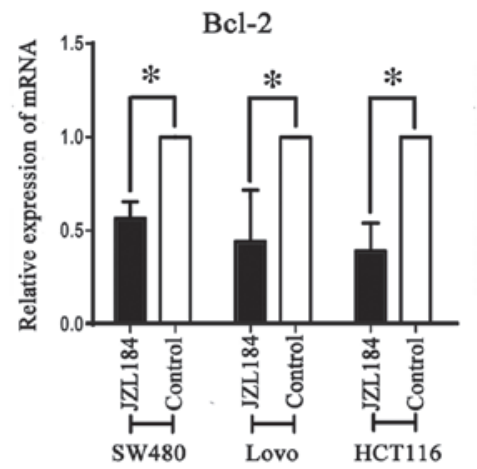

$\mathbf{C}$

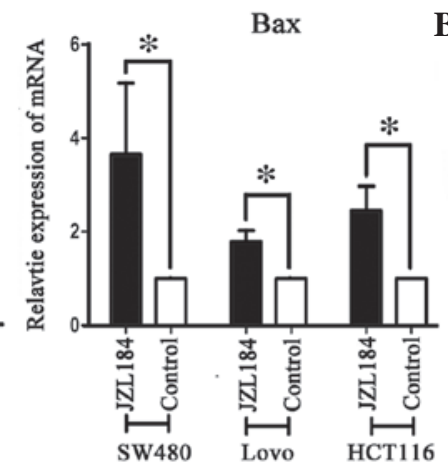

D
B

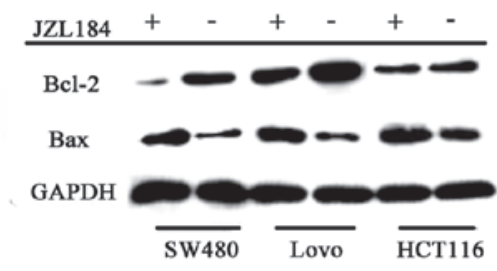

$\operatorname{Bax}$
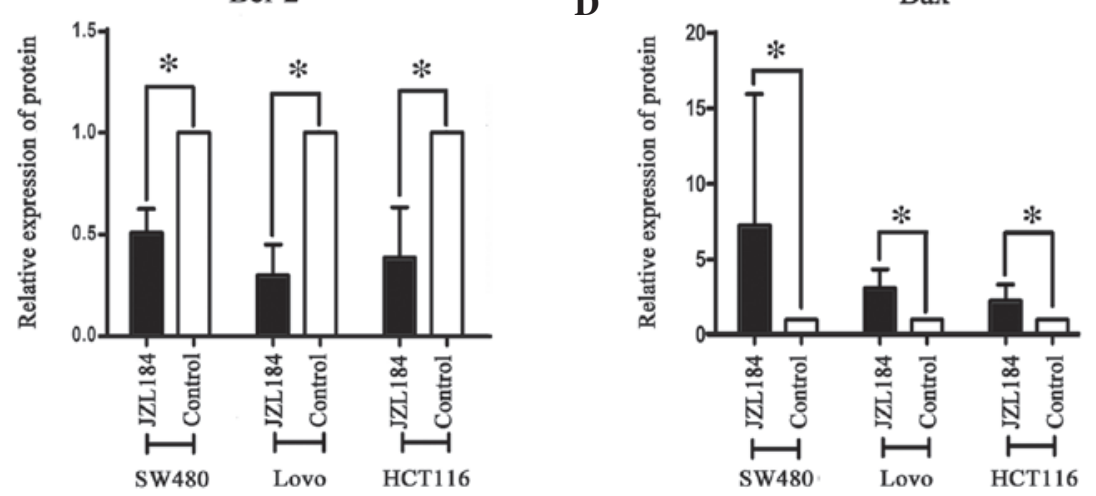

Figure 3. JZL184 regulated the expression of Bcl-2 and Bax. (A) mRNA expression levels of Bcl-2 decreased with JZL184 administration, whereas Bax expression was increased, as compared with the control group. (B-D) As assessed by western blotting, the protein expression levels of Bcl-2 were markedly suppressed by JZL184 intervention in the three colorectal cancer cell lines, and the protein expression levels of Bax were increased, as compared with the control group. Data are presented as the mean \pm standard deviation. "P<0.05. Bcl-2, B-cell lymphoma 2; Bax, Bcl-2-associated X protein; GAPDH, glyceraldehyde-3-phosphate dehydrogenase. 

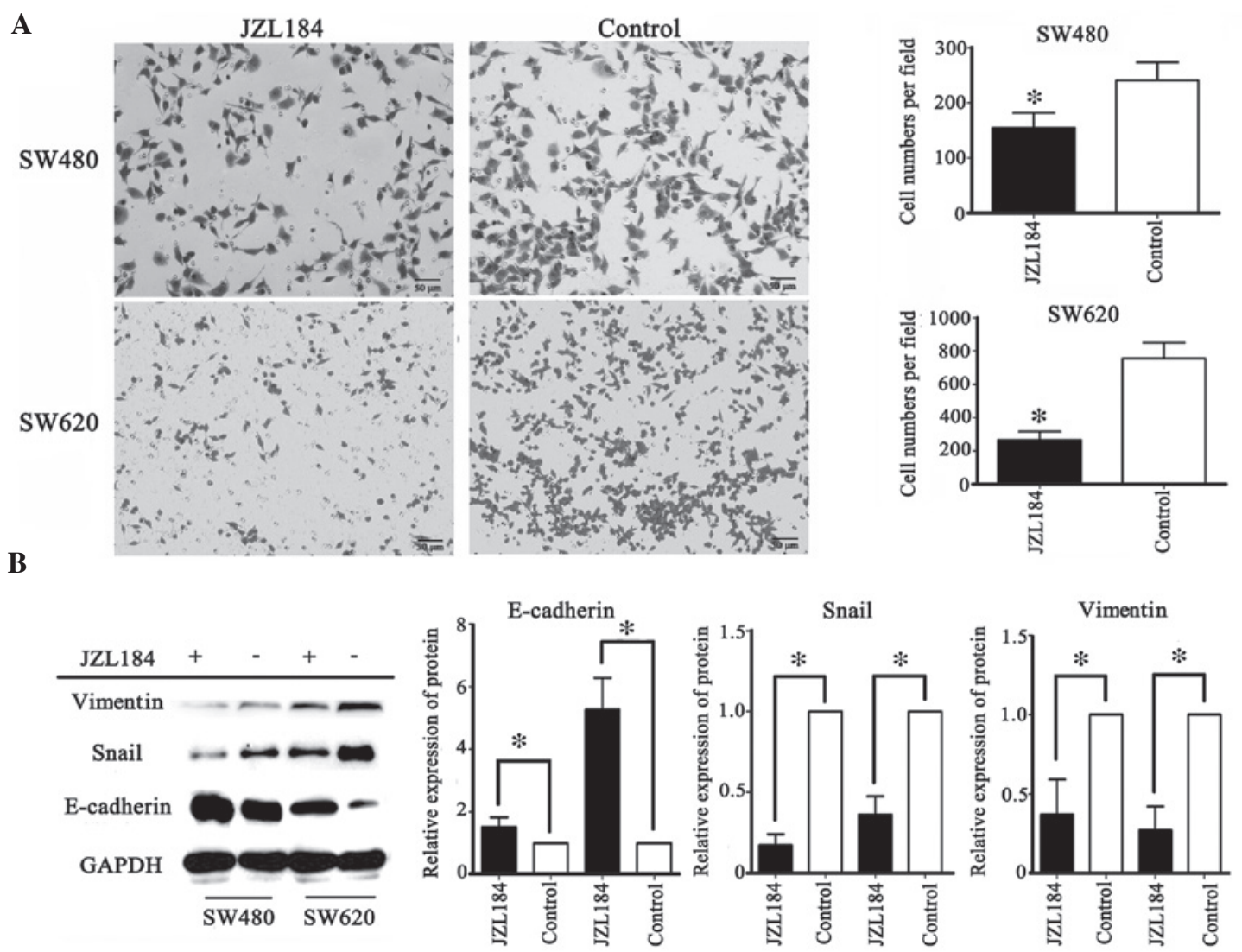

Figure 4. JZL184 inhibited the migration of colorectal cancer cell lines and the association between MAGL and EMT. (A) Migratory ability was assessed by Transwell assay. Compared with the control group, the number of cells was decreased in the colorectal cancer cell lines that exhibited different levels of aggressiveness. Scale bar $=50 \mu \mathrm{m} .{ }^{*} \mathrm{P}<0.05$, vs. the control group. (B) Western blotting was used to detect the protein expression levels of EMT markers, following administration of JZL184, E-cadherin protein expression levels were increased, and vimentin and Snail expression levels were decreased in colorectal cancer cell lines. Data are presented as the mean \pm standard deviation. ${ }^{*} \mathrm{P}<0.05$. EMT, epithelial-mesenchymal transition; MAGL, monoacylglycerol lipase; Snail, zinc finger protein SNAI1; GAPDH, glyceraldehyde-3-phosphate dehydrogenase.

tion of SW480 and SW620 cells was significantly decreased. Compared with the SW480 cells, the suppression of SW620 cell migration was markedly increased (Fig. 4A).

JZL184 inhibits EMT in colorectal cancer cell lines. EMT in epithelial tumor cells is closely associated with the process of tumor cell metastasis. In the present study, migration was observed to be inhibited by JZL184 in colorectal cancer cell lines; therefore, the protein expression levels of EMT markers were detected by western blotting. Following JZL184 administration, the SW480 and SW620 colorectal cancer cell lines exhibited significantly increased E-cadherin protein expression and decreased vimentin and Snail protein expression (Fig. 4B), thus indicating that MAGL may regulate migration via EMT.

\section{Discussion}

In a tumor-bearing state, endogenous fatty acid hydrolysis and oxidation are increased, triglyceride conversion and plasma FFA levels are increased, and utilization of exogenous fat is reduced (21). Previous studies regarding MAGL have focused on the role of the nervous system endocannabinoid signals in peripheral tissues and the central nervous system. These have demonstrated that cannabinoids inhibit cancer cell proliferation, induce tumor cell apoptosis and influence tumor angiogenesis (22-24). Nomura et al (7) demonstrated that MAGL may regulate the fatty acid metabolism network via the MAGL-FFA pathway, resulting in an increase in various lipid signaling molecules, including lysophosphatidylcholine and lysophosphatidic acid ethanolamine, and particularly lysophosphatidic acid and prostaglandin $\mathrm{E}_{2}\left(\mathrm{PGE}_{2}\right)$, which have been indicated to be closely associated with tumor proliferation and metastasis. Nomura et al (8) also demonstrated that in androgen-dependent prostate cancer, MAGL influences tumor proliferation and metastasis via the cannabinoid system and fatty acid network.

The present study demonstrated that tumor cell proliferation was reduced and apoptosis increased in response to MAGL inhibition. Furthermore, treatment with JZL184 and 5 -fluorouracil induced greater cell apoptosis than 5-fluorouracil alone in colorectal cancer cell lines. These results suggested that JZL184 may decrease tumor growth and enhance sensitivity to chemotherapy. In addition, Bcl-2 and Bax mRNA and protein expression levels were altered by JZL184 administration. This may suggest that MAGL regulates Bcl-2 and Bax protein expression levels to affect cancer cell activity. Ye et al (11) demonstrated that MAGL expression was positively correlated with BMI values, and individuals with a BMI $>30$ had higher levels of MAGL. Inhibition of MAGL activity with corresponding small interfering RNA was able to reduce the protein expression levels of cyclin D1 and Bcl-2, decrease 
tumor proliferation, induce apoptosis of tumor cells, and result in cell cycle arrest. The Bcl-2 protein family comprises key regulators of apoptosis, and alterations to protein expression affects the apoptosis of cells with DNA damage or cell cycle abnormalities, thus influencing the apoptosis of tumor cells. Bcl-2 and Bax exert an inhibitory and promoting effect on apoptosis, respectively (25). Increasing Bax protein expression increases cell sensitivity and apoptosis. A decrease in Bax protein expression reduces its preventative effects on $\mathrm{Bcl}-2$, thus promoting cell survival $(26,27)$. By inhibiting MAGL with JZL184, the present study demonstrated that MAGL may regulate Bcl-2 protein expression, thus promoting the effects of Bcl-2 and reducing Bax protein expression levels, resulting in anti-apoptotic effects in colorectal cancer cells.

EMT-associated proteins are important in the process of EMT (28). EMT results in tumor cell migration and increased invasive ability. The epithelial marker, E-cadherin, is decreased during EMT; however, vimentin and Snail are increased (29). It has previously been demonstrated that vimentin (22) is associated with the degree of tumor malignancy. EMT regulates the behavior of tumor cells (29), and the abnormal expression of E-cadherin is a key marker of the EMT process. Joyce et al (30) reported that MAGL may act as an EMT gene expression marker. Hu et al (31) investigated the association between nasopharyngeal carcinoma (NPC) and MAGL, and observed that MAGL promoted the metastasis of NPC by affecting EMT. These previous studies suggested that MAGL may be closely associated with colorectal cancer and the EMT process. In order to investigate the role of MAGL in the EMT of colorectal cancer cell lines, cells with various metastatic potentials were selected. The migration of colorectal cancer cells was significantly suppressed by JZL184 intervention, particularly in SW620 cell, as compared with SW480 cells. These results suggested that JZL184 interrupted cell migration, and as the degree of malignancy increased, the tumor cells were more sensitive to the effects of JZL184. To elucidate a possible mechanism underlying the effects of JZL184 on the colorectal cancer cell lines, the expression levels of EMT markers were detected. The present study observed that JZL184 increased the protein expression levels of E-cadherin, and decreased the protein expression levels of Snail and vimentin. These results indicated that the MAGL inhibitor, JZL184, decreased migration of colorectal cancer lines via influencing EMT, and the inhibitory effects was enhanced depending on the metastatic potential of the colorectal cancer cell line. MAGL, as a colorectal EMT marker, increases the invasive and metastatic potential of tumor cells, possibly via regulation of tumor cell EMT. In addition, metabolic products in the regulation of MAGL, including $\mathrm{PGE}_{2}$ (32), have been demonstrated to be associated with tumor cell EMT, and the endocannabinoid system is also associated with the process of EMT (5). However, whether MAGL regulates the EMT of colorectal cancer via the fatty acid network or endocannabinoids remains to be elucidated.

In conclusion, the MAGL inhibitor, JZL184, induced apoptosis by regulating the expression of $\mathrm{Bcl}-2$ and $\mathrm{Bax}$ in colorectal cancer cells. In addition, JZL184 increased the sensitivity of colorectal cancer cells to 5-fluorouracil, decreased migration of the tumor cells, and regulated the expression of EMT markers. These results suggested that MAGL may be considered a potential therapeutic target, and JZL184 may be a possible therapeutic agent for the treatment of colorectal cancer. Further research regarding the role of MAGL in colorectal cancer is required, which may elucidate the mechanisms underlying how JZL184 and MALG regulate proliferation and metastasis of colorectal cancer cells.

\section{Acknowledgements}

The present study was supported by the National Natural Science Foundation of China (grant no. 81172294).

\section{References}

1. King AR, Lodola A, Carmi C, Fu J, Mor M and Piomelli D: A critical cysteine residue in monoacylglycerol lipase is targeted by a new class of isothiazolinone-based enzyme inhibitors. Br J Pharmacol 157: 974-983, 2009.

2. Blankman JL, Simon GM and Cravatt BF: A comprehensive profile of brain enzymes that hydrolyze the endocannabinoid 2-arachidonoylglycerol. Chem Biol 14: 1347-1356, 2007.

3. Pisanti S, Picardi P, D'Alessandro A, Laezza C and Bifulco M: The endocannabinoid signaling system in cancer. Trends Pharmacol Sci 34: 273-282, 2013.

4. Hermanson DJ and Marnett LJ: Cannabinoids, endocannabinoids, and cancer. Cancer Metastasis Rev 30: 599-612, 2011.

5. Panikashvili D, Simeonidou C, Ben-Shabat S, Hanus L, Breuer A, Mechoulam R and Shohami E: An endogenous cannabinoid (2-AG) is neuroprotective after brain injury. Nature 413: 527-531, 2001.

6. Long JZ, Li W, Booker L, Burston JJ, Kinsey SG, Schlosburg JE, Pavón FJ, Serrano AM, Selley DE, Parsons LH, et al: Selective blockade of 2-arachidonoylglycerol hydrolysis produces cannabinoid behavioral effects. Nat Chem Biol 5: 37-44, 2009.

7. Nomura DK, Long JZ, Niessen S, Hoover HS, Ng SW and Cravatt BF: Monoacylglycerol lipase regulates a fatty acid network that promotes cancer pathogenesis. Cell 140: 49-61, 2010.

8. Nomura DK, Lombardi DP, Chang JW, Niessen S, Ward AM, Long JZ, Hoover HH and Cravatt BF: Monoacylglycerol lipase exerts dual control over endocannabinoid and fatty acid pathways to support prostate cancer. Chem Biol 18: 846-856, 2011.

9. Zlobec I and Lugli A: Epithelial mesenchymal transition and tumor budding in aggressive colorectal cancer: Tumor budding as oncotarget. Oncotarget 1: 651-661, 2010.

10. Joyce T, Cantarella D, Isella C, Medico E and Pintzas A: A molecular signature for epithelial to mesenchymal transition in a human colon cancer cell system is revealed by large-scale microarray analysis. Clin Exp Metastasis 26: 569-587, 2009.

11. Ye L, Zhang B, Seviour EG, Tao KX, Liu XH, Ling Y, Chen JY and Wang GB: Monoacylglycerol lipase (MAGL) knockdown inhibits tumor cells growth in colorectal cancer. Cancer Lett 307: 6-17, 2011.

12. Hector S and Prehn JH: Apoptosis signaling proteins as prognostic biomarkers in colorectal cancer: A review. Biochim Biophys Acta 1795: 117-129, 2009.

13. Prabhudesai SG, Rekhraj S, Roberts G, Darzi AW and Ziprin P: Apoptosis and chemo-resistance in colorectal cancer. J Surg Oncol 96: 77-88, 2007.

14. Oltvai ZN, Milliman CL and Korsmeyer SJ: Bcl-2 heterodimerizes in vivo with a conserved homolog, Bax, that accelerates programmed cell death. Cell 74: 609-619, 1993.

15. O'Leary DP, Bhatt L, Woolley JF, Gough DR, Wang JH, Cotter TG and Redmond HP: TLR-4 signalling accelerates colon cancer cell adhesion via NF- $\mathrm{BB}$ mediated transcriptional up-regulation of Nox-1. PLoS One 7: e44176, 2012.

16. Bai J, Chen J, Ma M, Cai M, Xu F, Wang G, Tao K and Shuai X: Inhibiting enhancer of zeste homolog 2 promotes cellular senescence in gastric cancer cells SGC-7901 by activation of p21 and p16. DNA Cell Biol 33: 337-344, 2014.

17. Leng Z, Tao K, Xia Q, Tan J, Yue Z, Chen J, Xi H, Li J and Zheng H: Krüppel-like factor 4 acts as an oncogene in colon cancer stem cell-enriched spheroid cells. PLoS One 8: e56082, 2013. 
18. Livak KJ and Schmittgen TD: Analysis of relative gene expression data using real-time quantitative PCR and the 2(-Delta Delta C(T)) Method. Methods 25: 402-408, 2001.

19. Croker BA, O'Donnell JA, Nowell CJ, Metcalf D, Dewson G, Campbell KJ, Rogers KL, Hu Y, Smyth GK, Zhang JG, et al: Fas-mediated neutrophil apoptosis is accelerated by Bid, Bak, and Bax and inhibited by Bcl-2 and Mcl-1. Proc Natl Acad Sci USA 108: 13135-13140, 2011.

20. Lee JG, McKinney KQ, Pavlopoulos AJ, Park JH and Hwang S: Identification of anti-metastatic drug and natural compound targets in isogenic colorectal cancer cells. J Proteomics 113: 326-336, 2015.

21. Schlosburg JE, Blankman JL, Long JZ, Nomura DK, Pan B, Kinsey SG, Nguyen PT, Ramesh D, Booker L, Burston JJ, et al: Chronic monoacylglycerol lipase blockade causes functional antagonism of the endocannabinoid system. Nat Neurosci 13: 1113-1119, 2010.

22. Gustafsson SB, Palmqvist R, Henriksson ML, Dahlin AM, Edin S, Jacobsson SO, Öberg $\AA$ and Fowler CJ: High tumour cannabinoid CB1 receptor immunoreactivity negatively impacts disease-specific survival in stage II microsatellite stable colorectal cancer. PLoS One 6: e23003, 2011.

23. Guzmán M: Cannabinoids: Potential anticancer agents. Nat Rev Cancer 3: 745-755, 2003.

24. Zeestraten EC, Benard A, Reimers MS, Schouten PC, Liefers GJ, van de Velde CJ and Kuppen PJ: The prognostic value of the apoptosis pathway in colorectal cancer: A review of the literature on biomarkers identified by immunohistochemistry. Biomark Cancer 5: 13-29, 2013.
25. Moreno A, Figueras A, Lloveras B, Escobedo A, Griera E, Sierra A and Fabra A: Apoptosis in ductal carcinoma in situ of the breast. Breast J 7: 245-248, 2001.

26. Choi BH, Kim W, Wang QC, Kim DC, Tan SN, Yong JW, Kim KT and Yoon HS: Kinetin riboside preferentially induces apoptosis by modulating Bcl-2 family proteins and caspase- 3 in cancer cells. Cancer Lett 261: 37-45, 2008.

27. Zhao S, Konopleva M, Cabreira-Hansen M, Xie Z, Hu W, Milella M, Estrov Z, Mills GB and Andreeff M: Inhibition of phosphatidylinositol 3-kinase dephosphorylates BAD and promotes apoptosis in myeloid leukemias. Leukemia 18: 267-275, 2004.

28. Kalluri $R$ and Weinberg RA: The basics of epithelial-mesenchymal transition. J Clin Invest 119: 1420-1428, 2009.

29. Satelli A and Li S: Vimentin in cancer and its potential as a molecular target for cancer therapy. Cell Mol Life Sci 68: 3033-3046, 2011.

30. Joyce T, Cantarella D, Isella C, Medico E and Pintzas A: A molecular signature for Epithelial to Mesenchymal transition in a human colon cancer cell system is revealed by large-scale microarray analysis. Clin Exp Metastasis 26: 569-587, 2009.

31. Hu WR, Lian YF, Peng LX, Lei JJ, Deng CC, Xu M, Feng QS, Chen LZ, Bei JX and Zeng YX: Monoacylglycerol lipase promotes metastases in nasopharyngeal carcinoma. Int J Clin Exp Pathol 7: 3704-3713, 2014.

32. Neil JR, Johnson KM, Nemenoff RA and Schiemann WP: Cox-2 inactivates Smad signaling and enhances EMT stimulated by TGF-beta through a PGE2-dependent mechanism. Carcinogenesis 29: 2227-2235, 2008. 